\title{
Desarrollo y capital social a la luz del pensamiento de Albert Hirschman: Sobre el arte de los traspasos y de las autosubversiones ${ }^{1}$
}

\author{
Javier Santiso
}

"Traspassing se emplea, a menudo, con sentido negativo en Estados Unidos, por ejemplo, en los carteles públicos en los que se ve escrito «No Traspassing», indicando una violación de la propiedad privada; pero desde mi perspectiva se reviste de un valor positivo: quiere decir superar las fronteras disciplinarias, pasar de una disciplina a otra sin rigidez. La última inversión de significado la hice con el término de «subversión», que normalmente es utilizado en la acepción negativa. ¿La subversión? ¡Una cosa terrible!».

Albert Hirschman. ${ }^{2}$

«Desarrollo y capital social a la luz del pensamiento de Albert Hirschman: sobre el arte de los traspasos y de las autosubversiones»

Traspasos y' autosubversiones: Hirschman convirtió estos ejercicios en un arte, un alegato que combina curiosidad y humildad intelectual. En un mundo acostumbrado a pensar y pensarse mediante modelos totalizadores, se llamen "teoría de la dependencia» 0 "consenso de Washington», en un continente donde se armaron y desarmaron tantos y tantos modelos ideológicos que pretendieron abrir (o mejor dicho forzar) la realidad concreta de los paises, la obra y la actitud intelectual de Hirschman constituyen una saludable y benéfica

\footnotetext{
- Por los comentarios y, las discusiones compartidas, asi como por los documentos transmitidos, quiero manifestar mi agradecimiento a Andrés Bajuk, Jesús del Río, Léo Harari, Guy Hermet, Bernardo Kliksberg, Rafael La Porta, Norbert Leckner, Florencio López de Silanes, Dani Rodrik, Alfredo Valladao, Romain Wacziarg y a todos los participantes en los foros organizados en Paris y Madrid. Un especial agradecimiento también a Enrique Iglesias, Andrés Bajuk y Léo Harari por la invitación ofrecida a utraspasar» fronteras y por haberme permitido prolongar con Albert Hirschman una conversación iniciada hace mas de cinco años en Berlin, y prolongada en Princeton en enero de 1999.

"Albeti Hirschman. Entrevista sobre su vida y obra", Desarrollo Económico, vol. $35, \mathrm{n}^{2} 140$, enero-marzo 1996, p. 658. Ver también el último libro de Hirschman, cuyo título es significativo de su defensa e ilustración de su peculiar curvatura intelectual y propensión a traspasar y autosubertir, Albert Hirschman, Crossing Boundaries, New York, Zone Books, 1998.
} 
invitación. Este no es, como veremos, el único mérito de su hazaña. De Chile a Brasil, de México a Argentina, su pasión por lo posible se contagió a más de uno. Muchos ministros, académicos y operadores de agencias internacionales no dejaron, en los últimos años, de saludar sus contribuciones. Igualmente, muchos de los conceptos desarrollados por Hirschman, su tríptico exit, voice and loyalty, la noción de efecto tinel y, sobre todo, su propensión posibilista, sus intentos portraspasar y autosubvertir las teorias (las suyas incluidas), los paradigmas y los modelos, todos los cubismos y minimalismos mentales que no dejan de nacer y renacer; constituyen saludables fuentes de inspiración e interpretación para repensar la nunca acabada buisqueda del clesarrollo. Por último, nociones como las de participación conunitaria o las de capital social, hoy en día en la agenda de las reflexiones, también se dejan apreciar; subvertir y autosubvertir a la luz de la obra de Hirschman.

En uno de sus más famosos ensayos, Isaiah Berlin sugería dividir los pensadores en dos categorías: los erizos y los zorros. ${ }^{3}$ Con esta original clasificación, el filósofo inglés pretendía ubicar a todos los intelectuales, inspirándose en un fragmento del poeta griego Arquíloco para quien «el zorro sabe muchas cosas, el erizo una sola pero grande».

Los erizos son aquellos que desarrollan una visión del mundo central, un sistema coherente mediante el cual analizan y piensan la totalidad de sus experiencias y pensamientos. Los zorros, por oposición, viven, piensan y actúan sin pretender ubicar sus vidas, sus reflexiones y sus acciones dentro de un sistema coherente y global, una visión del mundo totalizadora. Sin duda alguna, dadas su obra y su vida, Albert Hirschmman se ubicaría en la familia de los zorros, un zorro inmensamente libre que no deja de correr, traspasando tanto las fronteras mentales como las reales, las intelectuales como las físicas, multiplicando las visiones del mundo, las subversiones y autosubversiones, intentando siempre navegar contra los vientos y las corrientes por muy fuertes que sean los temporales ideológicos.

JIsaiah Berlin, "Le hérisson et le renard", en Berlin, Les penseurs russes, Paris, Albin Michel, 1984, pp. 57118 (traducido de Isaiah Berlin, Russian thinkers, Londres, The Hogarth Press, 1979). 
En los años treinta, huyendo de la Alemania nazi, multiplica los traspasos de fronteras reales, recorriendo prácticamente toda Europa realizando sus estudios en Francia (soñando entonces con entrar en Sciences Po y finalmente ingresando en una business school francesa ${ }^{4}$ ), en Inglaterra (LSE), antes de irse a Italia (Trieste) y luchar contra los fascistas primero en el ejército francés y más tarde en el americano. Cruza después el Atlántico para ubicarse en Estados Unidos donde desarrollará una brillante carrera universitaria en Columbia, Yale, Harvard y Princeton Incansable peregrino, en los años cincuenta y sesenta sigue traspasando fronteras; vive en Colombia unos años, país donde regresará en varias ocasiones y que le inspirará su primer gran ensayo sobre economía del desarrollo, un ensayo magistral que sigue siendo hoy en día un clásico de la literatura.

Sus experiencias latinoamericanas, en Chile, en Brasil, en Perú, en Uruguay, en Ecuador, en todo el continente, no dejaron de multiplicarse convirtiendo a Hirschman en el más europeo de los latinoamericanistas norteamericanos. Con el tiempo, sus reflexiones también fueron viajando rumbo a otras geografías mentales, traspasando su obra progresivamente las fronteras disciplinarias, zarpando de las ciencias económicas a las ciencias morales y políticas. A partir de los años setenta, después de haber asentado uno de los mayores intentos de subversión de las teorías del desarrollo entonces vigentes y dedicado a uno de los más sorprendentes intentos de autosubversión de sus propias teorías, Hirschman prolongó la aventura hacia otras direcciones. Sus nuevas peregrinaciones le llevarán a formular ideas originales no sólo sobre la economía del desarrollo sino también sobre la historia de las ideas, los vínculos entre economía y política y, en sus últimos ensayos, sobre el arte, la alegría y el significado (no sólo económico sino también Político, moral y social) de compartir un banquete.

Traspasos y autosubversiones: Hirschman convirtió estos ejercicios en un arte, un alegato que combina curiosidad y humildad intelectual. Cuando, por ejemplo, desarrolla una crítica de la escuela de la dependencia, lo hace a partir de una autocrítica de sus propias tesis defendidas anteriormente. ${ }^{5}$ Sus reitera-

- Como confesará tiemamente y con sentido de humor el propio Hirschman, al recibir el doctorado honoris causas de Sciences Po en abril de 1989, "you will now understand why today's ceremony has a special, sweet flavor for me - sweet as revenge can be After fifty-six years and a number of most unlikely detours, the doors of Sciences Po have finally swung open for me - a fairly tale come true My heartfelt thanks for this happy endn. Albert Hirschman, 1995, op. Cit., p. 115. 
dos intentos por escapar a toda clasificación, por no encerrarse en un paradigma globalizador, llave maestra que abriría el entendimiento de toda la realidad política, económica y social, son hoy en día saludados tanto por los académicos como por los operadores del desarrollo. Sin embargo el zorro sigue corriendo, Hirschman sigue rechazando los intentos de canonización o de reducción de su obra a una «gran idea central», aunque esa gran idea fuese la del rechazo de toda gran idea. ${ }^{6}$ En un mundo acostumbrado a pensar y pensarse mediante modelos totalizadores, se llamen «teoría de la dependencia» o consenso de Washington» (modelos para armar, como hubiese dicho Cortazar), en un continente donde se armaron y desarmaron tantos y tantos modelos ideológicos que pretendieron abrir (o mejor dicho forzar) la realidad concreta de los países, la obra y la actitud intelectual de Hirschman constituyen, no cabe duda, una saludable y benéfica invitación.

Este no es, como veremos, el único mérito de su hazaña. De Chile a Brasil, de México a Argentina, su pasión por lo posible se contagió a más de uno. Muchos ministros, académicos y operadores de agencias internacionales no dejaron, en los últimos años, de saludar sus contribuciones. Igualmente, como veremos en este trabajo, muchos de los conceptos desarrollados por Hirschman, su tríptico exit, voice and loyalty, la noción de efecto tínel y, sobre todo, su propensión posibilista, sus intentos por traspasar y autosubvertir las teorías (las suyas incluidas), los paradigmas y los modelos, todos los cubismos y minimalismos mentales que no dejan de nacer y renacer, constituyen saludables fuentes de inspiración e interpretación para repensar la nunca acabada búsqueda del desarrollo. Por último, como veremos también más adelante, nociones como las de participación comunitaria o las de capital social, hoy en día en la agenda de las reflexiones, también se dejan apreciar, subvertir y autosubvertir a la luz de la obra de Hirschman.

s Ver Albert Hirschman, "A dissenter's confession: The strategy of economic development revisited», en Hirschman, Rival views of market society, and other recent essays, New York, Viking, 1986; Albert Hirschman, "Beyond asymetry: critical notes en myself as a young man and some other old friends", International Organization, vol, 32, invierno 1978, pp. 45-50; y anteriormente Albert Hirschman "The poltical economy of import-substituting industrialization in Latin American, The Quarterly Journal of Economics, $\mathrm{n}^{2} 82$, febrero de 1968, pp. 2-32; publicado nuevamente en Albert Hirschman, A bias for hope:. Essays on development in Latin America, New Haven y Londres, Yale University Press, 1971, pp. 85-123.

- Ver en ese sentido el articulo de Michael McPherson, «The social scientist as constructive skeptic: On Hirschman's role", en Alejandro Foxley, Michacel McPherson y Guillermo O'Donnell, eds., Developmept, democracy and the art of trespassing: essays in honor of Alberio. Hirschman, Norte Dame, University of Notre Dame Press, 1986, pp. 305-316; y la respuesta de Hirschman, «A propensity to self-subversion", en Foxley et al. 1986 , op. cit., pp. $277 \cdot 283$. 


\section{Journeys from economics to politics and beyond}

La obra intelectual de Hirschman, desde el punto de vista de la historia de las ideas y de la economía del desarrollo, presenta una doble originalidad. Fue a la vez una obra central y marginal. Central porque sus reflexiones sobre la economía del desarrollo, desde la publicación de The strategy en 1958 hasta A bias for hope, en 1971, pasando por Journeys en 1963, sus reflexiones sobre el unbalance growth, sus conceptos de backwardy forward linkages, se convirtieron en ejes ineludibles de las discusiones sobre el desarrollo económico. ${ }^{7}$ Marginal porque en el mainstream de su disciplina de origen, la economía, Hirschrnan se convirtió en una voz apreciada pero marginada, fuera de juego (es decir fuera de la teoría de los juegos). Se quedó al margen de los senderos que se convirtieron con el tiempo en autovías para los economistas, la econometría, la formalización y el paradigma del actor racional. ${ }^{8}$

Sin embargo, y al contrario que la derrota que sufrió la economía del desarrollo, como señala Krugman, ${ }^{9}$ una derrota no tanto empírica o ideológica sino mas bien metodológica (al haber quedado esa rama de la economía dominada por un estilo discursivo no matematizado a un momento en el cual se aceleraba la formalización en todas las otras ramas), Hirschman asumió plenamente su exilio intelectual. Su marginación disciplinaria, similar a la de algunos otros economistas del desarrollo como Myrdal por ejemplo, fue voluntaria: Hirschman sencillamente dimitió, dejó de correr por la autovía (por la que en su momento circuló), se apartó para salir a pasear por otros senderos y seguir disfrutando de paisajes, cierto menos allanados que los de las formalizaciones matemáticas, Pero mucho más ricos en colores, matizando lo económico con lo político y lo moral y viceversa.

\footnotetext{
7 Ver Albert Hirschman, The strategy, of economic development, New Haven, Yale University, 1958 ; Albert Hirschman, Journeys toward progress: studies in economic polcy-making in Latin American, New York, Twentieth Century Fund, 1963; Albert Hirschman, A bias for hope: essays on development and Latin American, New Haven, Yale University Press, 1991.

- Para un análisis epistemológico de esta evolución hacia una normalización creciente, ver Donald McCloskey, Knowledge and persuasion in economics, Cambridge y Nueva 'York, Cambridge University Press, 1994. "Ver Paul Krugman, "The fall and rise of development economics", in Lloyd Rodwin y Donald Schön, eds., Rethinknig the development experience, Essays provoked by the work of Albert o. Hirschman, Washington, DC y Cambridge, Mass., The Brookiings Institution y The Lincoln Institute of Land Policy, 1994, pp. 39-58, Ver tambiēn Paul Krugman, "Towards a counter-counterrevolution in development theory", Proceedings of the World Bank Annual Conference on Development Economics, Washington, DC, The World Bank, 1992, pp. 1538 ; y los ensayos del propio Hirschman sobre la evolución de la economía del desarrollo, en particular, Albert Hirschman, "The rise and decline of development economics", in Hirschman, Essays in trespassing: economics to politics and beyond, New York y Cambridge, Cambridge University Press, 1981, pp. 1-24.
} 
Regresando a los orígines de la economía política, al Adam Smith anterior a The Wealth of Nations, el de The Theory on Moral Sentiments, ${ }^{10} \mathrm{Hirschman}$ no deja de insistir en su obra en los cambios de preferencias de los individuos, auscultando sus pasiones y sus intereses, ${ }^{11}$ sus propensiones a movilizarse por causas comunes a emprender acciones colectivas o por contra retirarse a la esfera privada. ${ }^{12}$ La topografía de las ciencias económicas tal como la dibuja, año tras año, la distribución del Premio Nobel, indica que este modelo del actor, que transcurre en toda la obra de Hirschman, esté quizás regresando a mejor fortuna (o menor olvido). Sea Ronald Coase y su teoría de los costos de transacción, Douglas North y su institucionalismo económico o Amartya Sen y su ética económica, todos estos economistas señalan un modelo del actor más cercano al defendido por Hirschman que al preferido por Becker. ${ }^{13}$ De igual modo, en el área de la economía política internacional o de la economía del desarrollo, están floreciendo trabajos que combinan esprit de géométrie esprit definesse, que «complican» diría Hirschman, el discurso de la economía, como, por ejemplo los de Dani Rodrik y de otros muchos más. ${ }^{14}$

${ }^{2}$ Adarn Smith, The theory of moral sentiments, Oxford, Clarendon Press, 1976 (1759). Como señala Ronald Coase, en uno de sus ensayos, it is wrong to believe that Adam Smith had as his view of man an abstraction, an economic rationally pursuing his self interest in a single minded wayn: "Adam Smith would not have thought sensible to treat man as a rational utility maximizer", Ronald Coase, "Adam Smith's view of mann, Journal of Law and Economics, vol. 19, octubre 1976. pp. 545.546. Curiosamente esta obra de Smith tuvo una forma y una difusión menores que The Wealth of nations. No existe, por ejemplo ninguna reedición disponible de la traducción de su ensayo desde 1860 , época en la cual se reedita la traducciôn realizada por Sophie de Grouchy, Marquise de Condorcet, en 1789.

"Albert Hirschman, The hetoric of reaction: perversity, futility, jeopardy, Cambridge, Mass, Harvard University Press, 1991. ${ }^{2}$ Albert Hirschman, Shitting involvements. Private interest and public action, Princeton, Princeton University, 1982. ${ }^{23}$ Amartya Sen, en particular, se explicó a lo largo de su abra sobre su concepción del actor económico criticando la visión neoclásica e utilitarista del actor como urational fools" en un artículo famoso, ver Amartya Sen, "Rational fools: a critique of the behavioral foundations of economic theory", Philosophy and Public Affairs, vol. 6, $\mathrm{n}^{2} 4$, verano 1977, pp. 317-344; y también su libro Amartya Sen, On Ethics and economics, Oxford, Brasil Blackwell, 1987. Para una visión más clásica del actor económico como, ver, por ejemplo el discurso de recepción del Premio Nobel de Gary Becker, «The economic way, of looking at behavior. The Nobel Lecturen, Essays in Public Policy, no 69, Hoover Institution, 1996; y el artículo de George Stigler y Gary Becker, "De gustibus non est disputandum", American Economic Review, vol: 67. marzo 1977, pp. $76 \cdot 89$.

14 ver, por ejemplo, los últimos ensayos de Dani Rodrik, The new, global economy and developing countries, Baltimore, Johns Hopkins University Press, 1998 ; o los trabajos reunidos en Federico Sturzenegger y Mariano Tommasi, eds., The political economy of economic reforms, Cambridge, Mass., MIT Press, 1998. 


\section{¿Del buen revolucionario al buen liberal?}

Lo que sí es seguro es que, durante la «década perdida», el trabajo de Hirschman cobró, en América Latina en particular, y en el mundo de los académicos y de los operadores del desarrollo en general, un cachet especial.

A partir de los ochenta, abundan las reverencias a su obra y a su trabajo, reverencias que en ningún caso son «totemizaciones» como señalan Alejandro Foxley, Michael McPherson y Guillermo O'Donnell en la introducción de su libro dedicado al pensamiento de Hirschman. De hecho cuando hubo totemizaciones, el propio Hirschman se esforzó en rehusarlas como ilustró en su conferencia presentada en el acto celebrado por el Banco Mundial, a principios de los ochenta, para homenajear a los «pioneros» del desarrollo. ${ }^{15}$ En abril de 1984, un congreso enteramente dedicado al ensayista es organizado en la Notre Dame University. Dos años más tarde, en noviembre de 1989, con el apoyo del Banco Interamericano de Desarrollo, otro congreso internacional tiene lugar en Buenos Aires, en el Instituto Torcuato di Tella, invitando a repensar la estrategia de desarrollo nuevamente a la luz del pensamiento de Hirschman. ${ }^{16}$ Otros más seguirán en la CEPAL, por ejemplo, o en el MIT donde se celebrará un importante encuentro de economistas para rediscutir las experiencias del desarrollo partiendo de su obra. ${ }^{17}$

Estos (re)encuentros son también, en ocasiones, testimonios de agradecimiento no sólo por la obra de un pensador sino también por el esfuerzo de un hombre que estuvo dedicando tiempo y energía, en los momentos más difíciles de las dictaduras militares, a apoyar a los demócratas latinoamericanos, intelectuales e instituciones de la región. Con el regreso de la democracia a América Latina, también regresaron muchos de estos intelectuales al mando político y económico. Por eso esta evolución, este «redescubrimiento» de

\footnotetext{
is Incluso y, sobre todo cuando esos mismos intentos de "totemizaciôn" apuntaban a interpretaciones abusivas de sus tesis. Ver Albert Hirschman, "A dissenter's confession: the strategy of economic development revisited", en Gerald Mejer y Dudley Seers, eds., Pioneers in development, New York, World Bank y Oxford University Pres5, 1984, pp. 104 Y s.

${ }^{16}$ Todas estas reuniones dieron lugar a ensayos recogidos en Alejandro Foxley, Michael McPherson y Guillermo O'Donnell eds., Development, democracy and the art of trespassing. Essays in honour of Albert Hirschman, Notre Dame, University Press, 1986; Simon Teitel, ed. Towards a new development strategy for Latin America: pathway from Hirschman's thought, Washington, DC, Inter-American Development, Bank y Johns Hopkins University, Press, 1992.

" Este encuentro dio lugar a una publicación señalada anteriormente, ver Lloyd Rodwin y Donald Schön, eds., 1994, op cit.
} 
Hirschman en los ochenta, no es sólo una curiosidad relevante desde el punto de vista de la historia de las ideas, sino que lo es también desde el punto de vista de la práctica de la economía política. Muchos de estos intelectuales, que tuvieron ocasiones de reiterar su agradecimiento y deuda hacia Hirschman llegaron a ser efectivamente ministros e incluso presidentes de su propia república nuevamente democrática a fines de los ochenta y en los noventa. Entre ellos, por ejemplo, figuran el ex-Ministro de Hacienda y Senador chileno Alejandro Foxley, o los brasileños Fernando Henrique Cardoso (hoy en día Presidente), Pedro Malan (Ministro de Hacienda) y José Serra (Ministro de Salud; este último fué asistente de investigación de Hirschman en el Institute for Advanced Study de Princeton mientras terminaba su doctorado en Cornell University) que participaron en alguno de los encuentros señalados anteriormente.

Durante la «década perdida» América Latina ganó algo muy valioso, El continente experimentó una conversión posibilista. ${ }^{18} \mathrm{~A}$ veces de manera voluntaria y deliberada, otras veces sin saberlo o sin quererlo, al igual que Monsieur Jourdain en la obra de Molière, las políticas económicas se volvieron eminentemente pragmáticas. Chile, quizás como ningún otro país, ilustra de manera idónea esta gran transformación latinoamericana, Este país no dejó de experimentar «un diluvio de paradigmas», pasando de una «revolución en libertad» a una «revolución socialista» y luego cambiando de nuevo a «una revolución liberal». A partir de los años ochenta, sin embargo, las políticas económicas se volvieron más pragmáticas, el país fue acumulando las «heterodoxias». Chile, al igual que otros países en la región, no país del paradigma del «buen revolucionario» al del «buen liberal»: lo que entra en crisis en los años ochenta es precisamente la política de lo imposible, la idea misma de fomentar políticas económicas pensadas y accionadas a partir de macro-paradigmas intangibles.

A principios de los ochenta, el país de los Chicago Boys, presentado como el antro del neoliberalismo en la región, nacionaliza sus bancos, ilustrando de manera patente la idea de Hirschman de unintended consequences de la acción humana y del posibilismo en materia de política económica. Como escribe Carlos Díaz Alejandro, refiriéndose a ese período, «the clearest example

\footnotetext{
${ }^{18}$ Ver, para una defensa e ilustración de esta tesis, Javier Santiso, De l'utopisme au possibilisme: une analyse temporelle des trajectoires mexicaines et chiliennes, 1970-1996, PhD Dissertation, Sciences Po, Paris, 1997.
} 
of this paradox is Chile, which, guided by able economists committed to laisse-faire, showed the world yet another road to a de facto socialized banking system. Argentina and Uruguay, show similar trends, which can be detected neatly in other developing countries». ${ }^{19}$ Años más tarde, cuando los Chicago Boys dejan el poder con la caída del régimen militar, los dirigentes chilenos, en vez de repudiar la herencia económica, la experiencia acumulada durante el régimen militar en materia de ingeniería y reforma económica, seguirán combinando privatizaciones y regulaciones, apertura a los flujos comerciales y de capitales y control de capitales (mediante el famoso sistema del encaje eliminando en 1998, cuando todos apuntaban hacia ese «modelo» en el cual había que inspirarse para frenar el contagio de las crisis financieras), ${ }^{20}$ matizando pues, de manera posibilista, el crecimiento con equidad.

\section{A passion for the possible}

Podríamos multiplicar los ejemplos de este giro posibilista que se dio y se está dando en la región, Como señala el propio Hirschman, en su último ensayo dedicado a la evolución de la economía del desarrollo en América Latina (ilustrando su propósito a partir de ejemplos argentinos, brasileños y chilenos), «in an earlier article, I talked about contrasting switches from one set of beliefs to another. This time I am concerned with a more fundamental, if less easily defined, shift from total confidence in the existence of a fundamental solution for social and economic problems to a more questioning, pragmatic attitude - from ideological certainty to more open-ended, eclectic, skeptical inquiry. ${ }^{21}$

Si la obra de Hirschman cobra nuevamente relevancia es precisamente porque algo muy profundo ocurrió en América Latina durante las últimas décadas. Con el desarme deológico, que culmina con el derrumbe del muro de

\footnotetext{
"Carlos Díaz-Alejandro, "Some unintended consequences of financial laissez-faire", in Foxley, McPherson y O'Donnell, 1986, op. Cit., pp. 91*113.

${ }^{20}$ Sobre estas políticas económicas en particular podemos consultar especialmente los estimulantes trabajos de Sebastian Edwards y Daniel Lederman, «The political economy of unilateral trade liberalization: the case of Chilew, National Bureau of Economic Research Working Paper, no $61^{\circ}$, abril 1998; y, sobre los controles de capitales en particular, Sebastian Edwards, winterest rate volatility, capital controls, and contagion", NBER Working Paper, n² 6756, octubre 1998.

"Albert Hirschman, "On the politica economy of Latin American development", Latin American Research Review, $n^{2} 22,1987, \mathrm{pp}, 7-36$.
} 
Berlín en 1989, también se puso en tela de juicio un cierto estilo cognitivo patente, en la reflexión y acción en favor del desarrollo principalmente en América Latina. Un estilo caracterizado por Hirschman en sus ensayos (a partir de sus observaciones concretas llevadas a cabo en Brasil, Colombia y Chile a fines de los cincuenta, principios de los sesenta) como rupturista, esencialmente centrado en vislumbrar la realidad a través del prisma de los paradigrmas; un estilo de economía política no incremental, de reiterados intentos y fracasos que tejieron un complejo de fracaso y propensión pesimista, una fracasomanía que se intentaba superar, en ocasiones, con escaladas ideológicas, mediante lo que Hirschman llamó la rage de vouloir conclure, intentos de acelerar el desarrollo a partir de «respuestas pseudocreativas», soluciones integradas, definitivas y rápidas, que dejaban de lado las posibles secuencias cumulativas, los aprendizajes de experiencias anteriores. ${ }^{22}$

La llave mágica que abre el paraíso del desarrollo no se encontró. Quizá porque durante muchos años se pretendió descubrir una única llave que abriese idénticamente todas las puertas. Se buscó aplicar a todos los países latinoamericanos, las mismas recetas de cocina, idénticamente saladas o azucaradas, en función de las modas culinarias del momento, de l'air du temps que se respiraba en algún lejano y norteño faro de occidente. Se pretendió servir a todos los países del continente los mismos platos, fuertemente ideologizados e inicialmente calentados en alguna lejana universidad norteamericana o europea. Platos recalentados, cabe señalarlo, en numerosas ocasiones, con éxito y astucia para los climas más tropicales o andinos de América Latina.

No existen secuencias de desarrollo idóneas, algunas son simplemente peores que otras, dependiendo de los contextos locales. No existen vínculos sistemáticos entre democracia política y desarrollo económico, ${ }^{23}$ ni leyes de

\footnotetext{
22 Ver, su ensayo magistral, Albert Hirschman, Problem solving and policy-making: A Latin American style?, in Hirschman, Journeys toward progress, 1963, op. cit., pp. 227-246.

"Ver, sobre este punto, las aclaraciones de Albert Hirschman, "The on-and-offconnection between political and economic progress", American Economic Review. Papers and proceeding Vol. 84, mayo 1994, pp. 343 348. Para una discusión a partir de datos más cuantitativos, también se pueden consultar los ensayos de Dani Rodrik, «Democracies and economic performance, Harvard University, Departmnet of Economics, Cambridge, Mass., 1998 (mimeo); Adam Przeworski y Fernando Limongi, "Modernization; theories and facts", Il'op-Id Polit@cs, val, 491 no 2, enero 1997, pp. 155-193; Rpberl Barro, <@ Democracy and growth. th 35, Journal of Economic Growth, vol, 1, 1996, pp. 1-27 asi como los importantes trabajos de Alberto Alesina, The poltical economic c)f higli and iow growth, in Boris Pieskovic y Josepli Stiglit7, eds, Annual Conference Deizelopi@ient Economics 1997, Washington DC, The Worid Baiik, 1998 ; de Jos TaN, ares y Romain Wacziarg, How democracy fosters gr@h, Ha@ard Universi'ty, Deparment of Economics, Cambridge, Mass., 1996 (mimeo) y, por último, los de Alberto Alesina y Roberto Percsti, i@ The potitical economy of growth : a critical survey of tire recent litterature, The World Bank Economic Review, vol. 8, o' 3, 1994, pp. 351-571.
} 
cambios globales, válidas para todos. Quizás más vale, añade Hirschman, prescindir de las supuestas secuencias ideales y optar por un reformismo no sólo cumulativo sino también adaptativo: «looking for uniform solutions to development problems invariably leads us astray; this is so for the imperatives of simultaneity and sequentiality alike, that is so for the insistence on integrated planning as well as for the injunction to postpone certain tasks in the name of one thing at a time. With this conclusion I can lay claim to at least one continuity in my thought: the refusal to define one best way. ${ }^{24}$

\section{A little more reverence for life}

Uno de los leitmotiv de Hirschman, desde Strategy, es que los individuos y las instituciones se convierten en actores del desarrollo si participan de manera activa, es decir no sólo reactiva sino también creativa. Para Hirschman lo esencial es el leaming by doing. Los logros como los errores son valiosos senderos de aprendizaje; los obstáculos del desarrollo pueden convertirse en vectores del desarrollo, existen blessing in disguise y unintended consequences que derivan de lo que se puede asemejar, en primer análisis, a un fracaso o a un obstáculo. ${ }^{25}$ De la misma manera, Hirschman pudo argumentar que, en algunos casos, el desarrollo se puede lograr en ausencia de objetivos predeterminados, en ausencia de un conocimiento exhaustivo de la manera de lograrlos. La ausencia misma de conocimiento puede convertirse en un blessing in disguise: si las instituciones o los individuos involucrados en proyectos de desarrollo fueran conscientes de todas las dificultades que deben afrontar, probablemente decidirían abandonar y no emprender el esfuerzo. Este el famoso principio de la hiding hand que propone en uno de sus ensayos, en referencia a la mano invisible de Smith. ${ }^{26}$

De ahí su reiterado interés y preferencia por los pequeños cambios y las transformaciones graduales - que sólo se vislumbran como tales porque nos hemos acostumbrado a vivir en un tiempo mundial que favorece las compa-

${ }^{24}$ Albert Hirschman, "The case against one thing at time», World Development, $n^{2} 18$, Agosto 19g0, pp. 1119-1122.

${ }^{25}$ Estas ideas quedaron desarrolladas en su introducción al posibilismo, ver Albert Hirschman, Introduction: poltical economics and possibilism, en Hirschman, 1971, pp. 1-37.

${ }^{26}$ Ver Aibert Hirschman, "The principle of the hiding hand», Public interest, vol. 2, invierno 1967, pp, 1-23. 
raciones instantáneas, reduce las distancias y comprime los tiempos (mecanismos que fomentan y amplifican de manera inédita la «fracasomanía», un cierto complejo de impotencia ante el trabajo por realizar, el «camino» por recorrer ${ }^{27}$ ). De ahí también su reiterada insistencia en la mejor aceptación y mayor consideración por las realidades y experiencias concretas. In all these matters, escribe Hirschman refiriéndose a la economía del desarrollo, I would suggest a little more reverence for life, a little less straitjacketing of the future, a little more allowance for the unexpected and a little less wishfull thinking». ${ }^{28}$

Ello conlleva una más amplia aceptación de quienes son los actores del cambio (los cuales no son sólo los «desarrolladores» sino también los desarrollados») y una mayor y mejor involucración de sus principales destinatarios. Los estudios derivados de experiencias concretas corroboran la mayor efectividad de los programas participativos. Como muestra un informe del Banco Mundial sobre 121 proyectos de dotación de agua potable a zonas rurales, en el caso de los proyectos con alta participación (21\% del total) la efectividad y el grado de logro de las metas llegaron a un tope de $81 \%$, mientras los proyectos con baja participación ( $21 \%$ del total), la efectividad y el grado de logro de las metas llegaron a un tope de $81 \%$, mientras los proyectos con baja participación ( $31 \%$ del total) sólo $3 \%$ tuvieron de efectividad ${ }^{29}$. La experiencia del presupuesto municipal participativo de Porto Alegre, que se convirtió en una referencia internacional, también confirma esta mayor efectividad de los proyectos participativos, incluso cuando abarcan amplios grupos humanos $(1,300,000$ personas en este caso). La participación comunitaria, tanto al nivel de la expresión de los problemas como en la selección de las prioridades y en la evaluación de los resultados, permitió una asignación de los recursos más eficiente. Así, entre 1989 y 1995, la cobertura de agua subió del $80 \%$ al 98\% mientras el sistema de alcantarillado se extendió del $46 \%$ al $74 \% .^{30}$

En el proyecto rural participativo de prevención en materia de salud, llevado a cabo en Ceará, en el nordeste de Brasil a partir de 1987, también se

\footnotetext{
"Ver Albert Hirschman, "Policy-making and policy analysis in Latin America: a return journey", in Hirschman, 1981, op, cit., pp. $142 \cdot 166$.

${ }^{28}$ Albert Hirschman, "The search for paradigms as a hindrance to understanding», in Hirschman, 1971, op. cit., $\mathrm{pp}, 354$.

${ }^{29}$ Ver los ejemplos señalados por Bernardo Kliksberg, "Seis tesis no convencionales sobre particpación", documento de trabajo presentado en el foro Banco Inter-Americano de Desarrollo / Sciences Po, "Cultura y Desarrollo", París, 9・10 de octubre de 1998.

${ }^{3}$ Sobre esta experiencia de democracia participativa, ver la tesis doctoral: de José Luiz Marques, "Démocratie profane: l'expriérience du budget participatif de Porto Alegre Brēsil, 1989-1996", PhD diss., Sciences Po, Pańs, 1997
} 
consiguieron resultados importantes; se redujo de $36 \%$ la mortandad infantil (de 102 por 1000 a 65 por 1000), la tasa de cobertura de la vacunación pasó del $25 \%$ al $90 \%$ de la población. ${ }^{31}$ Por último, en el proyecto de Villa El Salvador en el Perú, se consiguieron levantar gracias a un importante esfuerzo comunitario gran parte de la infraestructura física en un vasto arenal ubicado en las afueras de Lima, y ello a lo largo de un gran esfuerzo que se prolongó durante dos décadas. En total se construyeron más de 38.000 viviendas, 60 locales comunitarios y otros tantos centros educativos, 41 unidades integradas de salud y educación pública, el analfabetismo se redujo drásticamente $(3,5 \%)$, situándose a un nivel muy por debajo de la media nacional. ${ }^{32}$

Otro aspecto importante señalado por Hirschman es que no sólo son los obstáculos objetivos al cambio los que impiden emprender senderos hacia el desarrollo sino, en muchas ocasiones, son los mismos obstáculos en las percepciones del cambio quienes lo impiden. ${ }^{33}$ La obsesión por las «reformas desde arriba», por las macro-reformas que permiten dar no un paso hacia adelante sino más bien un salto, un brinco majestuoso, favorece las decepciones. En algunos casos porque las metas son difícilmente alcanzables; en otros porque el deslumbrante brinco anunciado se convierte en una rotunda caída o porque el arranque inicial queda atrapado en la telaraña de impedimentos que no se vislumbraron. Dicha propensión impone un velo cognitivo que enmascara, o mejor dicho contribuye a encubrir, las experiencias y las posibilidades no sólo de «reformas desde abajo», de aprendizajes posibles a partir de las experiencias concretas. Por ello es imprescindible no sólo conocer experiencias de desarrollo exitosas sino también darlas a conocer; apuntar posibilidades y, quizás con ellas, contribuir a autesubvertir algunas de nuestras más enraizadas certidumbres.

Estas consideraciones tienen algunas consecuencias prácticas. Primero, involucrar de manera dinámica a los destinatarios de los programas de desa-

\footnotetext{
${ }^{31}$ Sobre esta experiencia ver el articulo de Judith Tendler y Sara Freedheim, "Bringing Hirschman back in: A case of bad government turned good", en Foxley, McPherson y O'Donnell, 1994 op, cit. pp. 176-209; Judith Tendler y Sara Freedheim, "Trust in a rent seeking worid: health and gonvernment transformed in world northest Brazil", World Development, 22 (12), pp. 1771-1791; y sobre todo el ensayo de Judith Tendler, Good governmet in the tropics, Baltimore, The Johns Hopkins University Press, 1997.

${ }^{32}$ Sobre esta experiencia en particular, ver la contribución de Carlos Franco, "La experiencia de Villa El Salvador: del arenal a los logros fundamentales a travês de un modelo social de avanzadas, en Bernardo Kliksberg eds., Pobreza: un tema impostergable. Nuevas respuestas a nivel mundial, México, Fondo de Cultura Económica, 1997, pp, 421-432.

33 Ver Albert Hirschman, "Underdevelopment, obstacles to the perception of change, and leadership", in Hirschman 197l, op, cit, pp. 328-34l.
} 
rrollo significa no sólo involucrarlos en el proceso de implementación sino también ex ante y expost, tanto en el proceso del diseño como en el del control y de la evaluación de dichos programas. Al igual que la samba o el tango el desarrollo no se aprende por correspondencia. Se necesita un learning by doing compartido, activo y retroactivo. De la misma manera el desarrollo es una danza que de ningún modo se baila sola. Se necesita una pareja, «el desarrolladon» y el «desarrollado», ambos aprenden haciendo, sobre sí, sobre el otro, sobre la acción, elaborada, llevada a cabo y evaluada en común, ambos aumentan así su capital de conocimiento acumulado, de savoir faire y faire savoir: Por último, el baile del desarrollo participativo implica también respetar, en el proceso mismo de la participación, sus tres tiempos: uno, elaboración de la agenda de prioridades; dos, gestión de la implementación de las secuencias; tres, evaluación de los logros, errores y omisiones. En cada uno de estos tres tiempo se requiere argumentación y negociación dentro de la pareja.

Las dificultades y los costos de los procesos participativos no son minimizables, en términos de tiempo por ejemplo, ya que extienden los plazos de decisiones y acciones. Sin embargo, sus virtudes resultan ser superiores no sólo desde el punto de vista de la eficiencia operativa, sino también en términos de ética económica ya que, como muestran los estudios recientes de Alesina y Boone, el desperdicio de la ayuda exterior no resulta ser leve cuando se multiplican los eslabones intermedios. ${ }^{34}$ Asegurar mayor participación por parte de los destinatarios finales es asegurar un desarrollo que sea realmente desarrollador para estos destinatarios y no para los intermediarios. Por otra parte, involucrar a las poblaciones no sólo desde el inicio del proyecto sino desde su concepción y elaboración permite también plantearse preguntas no sólo sobre el impacto económico sino también político y social de dicho proyecto: ¿Cómo alterará la ayuda externa el equilibrio político local? ¿Cómo afectará el capital social de los individuos? ¿Cómo consolidará o, al contrario, perjudicará la cohesión social de la comunidad?

En segundo lugar, dar a conocer las experiencias exitosas permite desplegar el abanico de posibilidades. Permite introducir un bias for hope y prestar mayor atención a los efectos no esperados que podrían haber quedado encubiertos, envueltos en el lienzo de las teorías y de los teoremas. Pero, sobre 
todo, dar a conocer las experiencias exitosas permite retroalimentar el proceso de aprendizaje. El conocimiento también es reconocimiento. No sólo es pertinente asegurar la publicidad externa sino también interna, hacia afuera como hacia adentro, hacia la comunidad de «desarrolladores». En muchos casos, se aprende haciendo, inventando e imitando. Dar a conocer es abrir otras posibilidades de aprendizaje mediante la emulación: conociendo otras experiencias se puede intentar imitarlas, repetirlas, se puede también inventar otras a partir de ellas, pero también se puede querer dar un paso más, superar sus logros.

Tanto el proyecto de Ceará, en el nordeste de Brasil, como el de Villa EI Salvador, en el Perú, permiten ilustrar las virtudes no sólo de la participación comunitaria sino también el de un conocimiento que es reconocimiento. En ambos casos se puso en marcha un proceso de aprendizaje participativo con componentes de incitación (por parte de los «desarrolladores») y, de imitación (por parte de los «desarrollados»). Pero, sobre todo, estas experiencias presentan componentes de emulación. Ambos proyectos recibieron laudos mundiales, el de Villa El Salvador fue galardonado por la UNESCO y por el Premio Príncipe de Asturias y el de Ceará recibió el Premio Maurice Paté de la UNICEF. Estos premios aseguraron conocimiento pero también reconocimiento, elevaron la autoestima individual y colectiva de todos los participantes reforzando su grado de implicación y estimulando otras experiencias. Un importante ejercicio de memoria viva queda por hacer para rescatar, documentar, revisar y difundir estas experiencias exitosas y aumentar el capital de conocimiento acumulado y compartido. Esto podría lograrse mediante, por ejemplo, un premio internacional, Como señala Bernardo Kliksberg, la existencia de un premio de esta índole en Canadá, motivó la presentación de unas 68 experiencias candidatas. De la misma manera se podría autosubervitir esta proposición asegurando igualmente un feedback de experiencias no exitosas, en las cuales lo que se premiaría sería la relevancia del análisis o de una posible solución del problema así puesto en evidencia.

\section{Capital social, concordia y discordia: Sobre las virtudes sociales del tiempo compartido y del tiempo conflictivo}

Uno de los temas centrales de los últimos ensayos de Hirschman es de las oscilaciones, en las acciones humanas, entre interés privado y acción públi- 
ca. En un primer ensayo, la división entre ambas esferas quedaba rotundamente afirmada. El propósito de Hirschman era de entender cómo se pasaba (traspasaba) de una esfera a la otra, cuáles eran en definitiva las motivaciones de estos cambios de preferencia. ${ }^{35}$ Más tarde, en ensayos ulteriores, fue matizando esta dicotomía para terminar auto-subvertiendo su tesis y mostrar como ambas esferas pueden fundirse y confundirse (en particular a partir del ejemplo de los banquetes públicos, como veremos mas adelante). De estos últimos ensayos se desprenden algunas consideraciones singularmente estimulantes para nutrir los debates contemporáneos sobre el capital social.

Hirschman no menciona directamente la noción de «capital social». ${ }^{36}$ Es sin embargo interesante subrayar que lo hace en una sola ocasión, y precisamente en un ensayo dedicado no a las virtudes de la cooperación sino todo lo contrario, a las virtudes del conflicto Social. ${ }^{37}$ En sus ensayos Putnam insiste sobre las virtudes del capital social como vínculos de confianza que tejen entre sí los individuos y como propensiones a la cooperación y convivencia pacífica. De la misma manera, los ensayos que corroboran la existencia de correlaciones entre la densidad de la vida asociativa, la confianza en sí y en los demás que tienen los individuos en las distintas sociedades, y la performance económica, se presentan en definitiva como una versión elaborada de la vieja tesis del doux commerce. ${ }^{38}$

Hirschman nos invita a una saludable autosubversión. Cierto, los vínculos de confianza, sean horizontales o verticales, inter-individuales o entre los individuos y las instituciones, son imprescindibles para consolidar la convivencia. De igual modo, como señalan Knack y Keefer, un alto nivel de confianza mutua permite a los individuos reducir el tiempo y el costo que implica

${ }^{35}$ Albert Hirschman, Shifing involvemets: Privale interest and public action, Princeton, Princeton University Press, 1982.

${ }^{36}$ De hecho es una nociōn dificil de atrapar en la red conceptual, ver Javier Santiso, "Le capital social comme capital temps: essai de franchissement", documento de trabajo preparado para el Foro BID/ Sciences Po, "Cuttura y Desarrollo", Paris, 9-10 octobre 1998.

${ }^{37}$ Ver su importante articulo, Albert Hirschman, "Social conflicts as pillars of democratic societies", in Hirschman, 1995 op. cit, pp, 231-248. En este trabajo menciona el libro de Robert Putnam, Making democracy work: civic traditions in moderm ltaly, Princeton, Princeton University Press, 1993. Esta idea de los conflictos como factores esenciales de la socialización se encuentra desarrollada también por otros autores como el sociólogo de principios de siglo, contemporáneo de Max Weber, Georg Simmel, Conflict and the web of group affiliations, Glencoe, III.,Free Press, 1995; y Marcel Gauchet, "Tocqueville, l'Amérique et nous", Libre, $\mathrm{n}^{0} 7,1980$, pp.116.117.

${ }^{38}$ Esta tesis fue ampliamente discutida por Hirschman en uno de sus más interesantes ensayos, Alber Hirschman, The passions and the interests: political arguments for capitalism before its triumph, Princeton, Princeton University Press, 1977. 
protegerse contra los riesgos que podrían resultar de las transacciones, comerciales o no comerciales, realizadas con los demás individuos. ${ }^{39}$ Pero la discordia, al igual que la concordia, también puede fomentar «capital social». El conflicto y la discrepancia, señala Hirschman quizás sean incluso más creadores de capital social que la propia cooperación pacífica señalada por Putman y sean además, en definitiva, marcadores más relevantes de la vitalidad y de la cohesión de una sociedad, The secret of vitality, escribe Hirschman, of pluralistic market society and of its ability to renew itself may lie in this conjunction (on both bargaining and arguing) and in the successive eruption of problems and crisis», «it cannot pretend to establish any permanent order or harmony; all it can aspire to accomplish is to «muddle through» from one conflict to the next». ${ }^{40}$

Entender los mecanismos de creación del capital social significa entonces prestar atención también a los conflictos. Obviamente se necesita para esto discriminar entre conflictos creadores y conflictos destructores de capital social. Combinar análisis cuantitativo, y análisis cualitativo para medir la relevancia de esa conflictualidad virtuosa. Evaluar no sólo la cantidad de conflictos sino también su calidad. Si bien se necesita analizar, tanto desde el punto de vista económico como político y moral, cómo la sociedades aseguran la convivialidad (comparando tiempo, por ejemplo, en un concierto de música o en un café, ${ }^{41}$ disfrutando en común una representación teatral o tomando un

39 Ver Stephen Knack y Philip Keefer, «Does social capital have an economic payoff? A cross-country investigation", The Quarterly, Journal of Economics, vol CXII, n은, noviembre 1997 pp. 1251・1288. Ver también Alberto Alesina y Romain Wacziarg, "The economics of civic trust, Harvard University, Deparmient of economics, septiembre 1998, mimeo.

$4^{\circ}$ Hirsschman, 1995, op, cit., pp, 243 y 244.

${ }^{4}$ Si bien abundan las sociologias de la convivialidad, y en particular la sociología de los cafés, los análisis en términos económicos son más escasos, a excepción notable de algunos economistas como por ejem. plo Tibor Scitovsky. En un libro admirable, Scitovsky señala que en las Ilamadas economías desarrolladas se vislumbra cierta atrofia de capital social si lo medimos como capital tiempo, es decir actividades de tiempo compartido. Los análisis de time-budget muestran efectivamente que entre 1934 y 1996 (datos señalados en su momento por Scitosky), en estos paises el tiempo dedicado a las comidas se redujo considerablemente, pasando de 107 a 70 minutos diarios. Igualmente el tiempo dedicado al paseo recreativo se redujo (de 22 a 1). En sus analisis Scitovsky destaca también la frecuencia y el tiempo dedicado por los franceses a los cafés. Ver Tibor Scitovsky, The joyless economy. An iquiry into human satisfaction and consumer dissastifaction, New York y Oxford, Oxford University Press, 1976 (en particular pp. 161-163 y 241-245). Estos trabajos son en definitiva invitaciones a ahondar los estudios comparativos, en términos de time-budget, sobre los comportamientos culturales en América Latina y, quizás, corroborar esa idea según la cual el "capital social», entendido como "capital tiempo" pueda que sea mejor distribuido en sociedades mas tradicionale, en las cuales predominan las actividades «holistas" sobre las actividades «individualista». Para una introducción al análisis económico de las artes, ver David Throsby, "The production and consumption of the Arts; a view of cultural economics", Journal of Economic Literature, $\mathrm{n}^{\mathrm{0}}$ 32, 1994, pp. 1-29. 
mate en un lugar público), también se necesita un mejor entendimiento de las instituciones y los mecanismos que regulan los conflictos sociales en América Latina. A nivel nacional, las instancias de regulación democráticas son un ejemplo en este sentido. Otras son las instancias jurídicas. Todas estas tienen que ver con la mencionada confianza vertical, entre los individuos y las instituciones nacionales. Convendría sin embargo prestar igualmente atención al nivel de confianza horizontal, entre los individuos, sobre todo en América Latina, donde, como subrayan los trabajos de Roberto da Matta, tienen específica relevancia no tanto los mecanismos de confianza vertical sino los de confianza horizontal, las relaciones interpersonales e informales (por oposición a las relaciones institucionalizadas o formalizada). ${ }^{42}$

De la misma manera, estas generalizaciones necesitan ser autosubvertidas a partir de análisis comparativos entre los diferentes países latinoamericanos. Los trabajos sobre los mecanismos de regulación de conflictos comerciales invitan a pensar que existen diferencias entre países como Argentina, donde los empresarios, al igual que los españoles o los franceses, prefieren resolver sus discrepancias fuera de los tribunales, y Colombia, donde los empresarios prefieran la resolución jurídica, al igual que los países escandinavos o anglosajones. ${ }^{43}$ No sólo a nivel nacional pero también local se necesita un mejor entendimiento de cómo quedan articuladas, asimiladas e implementadas las diferentes normas de convivencia que regulan discordias y concordias, que contribuyen a crear o destruir el famoso «capital social».

Si bien los economistas admiten la importancia del «civismo» de la «confianza» o de la adhesión a «normas éticas» compartidas para que la economía funcione de manera eficiente, resulta más problemático entender que ocurre cuando se activan esos inputs. El modelo de learning by doing, propuesto por Hirschman, inspirado por Arrow, ${ }^{44}$ permite reconsiderar este aspecto e insistir precisamente sobre el proceso acumulativo que se desencadena cuando se activa ese recurso o capacidad peculiar denominado «capital social».

Al igual que el capital físico, económico y financiero, al igual que el capital humano, el capital social se crea y se destruye, aumenta y disminuye.

\footnotetext{
${ }^{42}$ Roberto da Matta, Canaval, bandits el héros. Ambiuités de la société bresilienne, Paris, Editions du Seuil, 1983. ${ }^{43}$ Sobre la relevancia de los sistemas jurídicos para la resolución de los conflictos empresariales, existen unos primeros trabajos comparativos llevados a cabo por Rafael la Porta y Forencio López de Silanes, "Capital markets and legal institutions", Harvard University Department of Economics, Agosto 1998, mimeo.

${ }^{44}$ Kenneth Arrow, "The economic implications of learning by doing, Review of economic Studies, vol. 29, 1962, pp. $255 \cdot 173$.
} 
Todas la sociedades, tanto las pobres como las ricas, las que carecen de mecanismos educativos eficientes como las que gozan de ellos, todas poseen ese capital peculiar pero que, a diferencia de otras formas de capital, aumenta con su uso (y al contrario disminuye con su no utilización). El «amon», el «civismo», la «confianza» o las «normas éticas», no son recursos limitados, no disminuyen con su uso, al contrario: la competencia es un recurso que aumenta cuando se utiliza, «El amor o el civismo, escribe Hirschman, no son recursos limitados o fijos como lo pueden ser otros factores de producción», «son recursos cuya disponibilidad, lejos de disminuir, aumenta con su empleo».45

Un ejemplo particularmente interesante de esto son los banquetes cuyas virtudes no sólo son alimentarias sino también políticas y sociales como subraya Hirschman en un inspirado artículo. ${ }^{46}$ Los banquetes le permiten autosubvertir su idea de la clara y rotunda diferenciación entre lo público y lo privado al mismo tiempo que subrayar la dinámica «virtuosa» que activan. Considerados tradicionalmente como actos privados, los banquetes desempeñaron y desempeñan en realidad funciones eminentemente públicas. Constituyen actos sociales eminentes, en los cuales importa a veces tanto saber lo que se come como saber con quién se come. Actos en los cuales se crea y recrea capital social, convivialidad entre los diferentes participantes que tienen virtudes no sólo «civilizadoras», como muestran los sociólogos, entre ellos Simmel y Elias, sino también virtudes eminentemente políticas. La experiencia de compartir comida es una experiencia frecuente, reiterada y de cierta duración, permite acostumbrarse a ver, hablar y reunirse en torno a una mesa, un asado, fuera o dentro de un local.

Es sobre todo una acción de tiempo compartido, una acción comunitaria por excelencia cuyos beneficios externos justificarían en sí mismos subversiones (al igual que las justificadas por Scitovsky, como recuerda Hirschman, en beneficio de las artes). De hecho en la Grecia antigua los banquetes, por ser considerados como la máxima manifestación del vínculo social, del vinculo público, eran subvencionados por las familias acomodadas, considerando la comida en común como una institución que manifestaba la permanen-

\footnotetext{
${ }^{45}$ Albert Hirschman, "Trois façons simples de compliquer le discours de l'économie politique, en Hirschman, Vers une économie politique élargie, París, Editions de Minuit, 1986, pp. 89・110.

${ }^{46}$ Albert Hirschman, "Mêler les pshères publique el privée: prendre la commensalité au sérieux, en Hirschman, La morale secrète de l'économist, París, Les Bellae Lettres, 1997, pp. 131-169.
} 
cia del poder político en la democracia. De modo similar, en los inicios de la República Francesa, los banquetes también tuvieron funciones políticas, testimonios de una ética de la convivialidad que permitía consolidar el sentimiento de pertenencia y eran lugares de aprendizaje de lo político. ${ }^{47}$

$\mathrm{Si}$ bien esto no es una invitación a subvencionar banquetes para crear y recrear «capital social» (como lo subraya Hirschman existen también banquetes que no fueron nada virtuosos, entre otros los celebrados por los fascistas alemanes), sí lo es para plantearse una última pregunta: ¿cómo asegurar mayor participación?

Queremos terminar ofreciendo un esquema interpretativo a partir de un tríptico elaborado a principios de los setenta en un libro famoso: Exit, voice and Loyalty.

\section{Capital social y participación: Una reinterpretación a partir del triptico hirschmaniano exit, voice and loyalty}

En este trabajo Hirschman se proponía entender cómo los sistemas económicos (las empresas) o políticos (los estados) afrontan la defección de sus clientes o de sus ciudadanos. Hirschman propone entender estas dinámicas a partir de tres respuestas posibles por parte de los clientes descontentos: la salida (el cliente o el ciudadano se va, deja de comprar el producto o de participar en su comunidad nacional de origen), la voz (el cliente protesta, manifiesta su descontento, el ciudadano se manifiesta, provoca huelgas) o, a pesar de todo, mantiene su lealtad (se queda con el producto, mantiene una existencia cívica). ${ }^{48}$

Este modelo interpretativo fue ampliamente utilizado y reutilizado, no sólo para explicar fenómenos económicos sino también políticos. Es, por

\footnotetext{
47 Sobre la Grecia antigua y el republicanismo francés, ver Pauline Schmitt Pantel, La cité au banquet, Histoire des repas publics dans les cités grecques, Rome, Ecole Française de Rome, 1992; Olivier hl, La fête rëpublicaine, Paris, Gallimard, 1996; y Olivier linl, «De bouche à oreille. Sur les pratiques de commensalité dans la tradition républicaine du cérémonial de table», Revue Française de Science Politique, vol, 48, $\mathrm{n}^{\circ}$ 3*4, junio-agosto 1998, pp. 387-407. Igualmente relevante es saber lo que se come, con quién pero también cómo se come, las formas de cocinar caracterizando diferentes tipos de sociedades como señaló el antropólogo lack Goody, Cooking cuisine and class. A study in comparative sociology, Cambridge, Cambridge University Press, 1982.

${ }^{48}$ Albert Hirschman, Exit, voice, and loyalty: responses to decline in firms organizations, and States, Carnbridge, Mass., Harvard University Press, 1970.
} 
ejemplo, sencillo, deshacerse de sus acciones en bolsa (exit), sobre todo cuando los accionistas no tienen influencia sobre el corporate governance, es decir no tienen la posibilidad de alzar la voz. El mismo modelo interpretativo permite aclarar los mecanismos de difusión de las crisis financieras internacionales como hemos sugerido. ${ }^{49}$ En un matrimonio, cuando existen facilidades de divorcio, la propensión al voice puede ser menor, se harán menos esfuerzos de comunicación y tentativas de reconciliación. El propio Hirschman nos dio así a partir de este tírptico una interpretación original de la caída del muro de Berlín, fenómeno que desencadenó un exit masivo de los sistemas comunistas. ${ }^{50}$ En este trabajo aprovechó también la ocasión para autosubvertir su tesis inicial mostrando, en particular, que en este caso el exit y el voice no son fenómenos estrictamente contradictorios, respuestas automáticamente excluyentes la una de la otra sino que, al contrario, respuestas adaptativas o reactivas que se pueden combinar e incluso reforzar mutuamente.

En el ámbito del desarrollo, esta grille d'analyse también se puede aplicar a muchos problemas que se vislumbran. El dilema es siempre el mismo: cómo, por ejemplo, fomentar lealtad cuando se empreden modelos participativos. Uno de los aspectos centrales consiste en favorecer la expresión (el voice) de los destinatarios tanto al nivel de la formulación de la gestión como de la evaluación de los programas participativos. De esta manera se limita el exil, las potencialidades de fracaso y de dimisión. La clave esta en la invención de un mecanismo que permita consolidar la lealtad, la confianza y por lo tanto asegurar mayor y mejor participación. Como señala Hirschman, favorecer el voice no es cosa sencilla, los costos del voice siendo normalmente superiores al del voice, en términos de tiempo, de esfuerzo, de dinero. Sólo en configuraciones donde aparecen fenómenos de alta royalty, el voice puede resultar más costoso que el exit, es decir cuando la salida implica desprenderse de la esencia de su propia identidad. El voice en todo caso es preferible al exit, Favorecer la voz en los programas participativos permite recaudar, a lo largo de

49 Javier Santiso, «Financjal Crisis» Political Economy and the Art of trespassing, International Political Science Review, 1999 (en prensa).

so Albert Hirschman, "Exit, voice, and the fate of the German Democratic Republic", World Politics, no 45 , enero 1993, pp. 173-202. Ver también, para otros ejemplos, Albert Hirschman, "Exit and voice: and expanding sphere of influence", en Hirschman, Rival views of market society and other recent essays, New York, Viking, 1986; y Albert Hirschman, "Exit, voice, and loyalty: further reflections and a survey of recent contributions", en Hirschman, Essays in trespassing: economics to politics and beyond, Cambridge, Mass., Cambridge University Press, 1981 pp. 213-235. 
los tres tiempos de la danza, mayor información sobre las necesidades reales de los destinatarios de los programas. También permite asegurar una mejor ejecución y evaluación del programa favoreciendo los feedback inmediatos.

Esta grille d'analise cobra peculiar relevancia para analizar más de un problema contemporáneo al que nos enfrentamos cuando hablamos de desarrollo. En un mundo globalizado, interconectado, en el cual las fronteras se diluyen, los desniveles de vida resaltan aún más: en los poblados más retirados de la reserva maya de Sian $\mathrm{Ka}$ 'an por ejemplo se sabe, vía algún satélite, que hay, un ailleurs. Las antenas parabólicas apuntan hacia él de manera continua e instantánea, Si bien, los fenómenos de «exit», de migraciones siempre fueron importantes, hoy en día el reto de la fuga de cerebros es todavía mayor. En el caso de América Latina, se pasó de una situación de recepción de emigrantes a una situación de salidas masivas en particular de individuos altamente cualificados, es decir $e l$ exit abarca las fuerzas vivas, un precioso capital humano y social de los países, en particular los de América Central donde el fenómeno del brain drain es patente.

Un estudio reciente del Fondo Monetario Internacional muestra así que los emigrantes hacia Estados Unidos de estos países tienden a ser más cualificados que el promedio nacional de su país de origen. De manera general, para los países de América Latina y del Caribe, las tasas de emigración altamente cualificada son elevadas, superiores al $10 \%$ en la mayoría de los casos y en algunos superiores al $50 \%$. El país con mayor fuga de cerebros es Guyana donde más de $70 \%$ de los individuos con educación superior (terciara) salieron hacia Estados Unidos, le siguen luego Trinidad y Tobago (60\%), El Salvador (26\%), Panamá (19,5\%), Nicaragua (19\%), Honduras (16\%), República Dominicana (14\%) y Guatemala (13,5\%). En países como México (10,5\%) o Colombia (6\%), las tasas alcanzadas también son significativas, delante, de las de Argentina (2\%), Chile (3,5\%) o Uruguay (41\%). ${ }^{51}$

En materia de educación, como señala el último informe del BD, se plantean una serie de dificultades reinterpretables a partir de esta lectura hirschmaniana en términos de exit, voice y loyaltjy. ${ }^{52}$ Las familias de altos ingresos, como muestran los estudios, evitan la educación pública, produciéndose una

\footnotetext{
s" Ver William Carington y Enrica Detragiache, "How big is the Brain Drain?", 1MF Working Paper, julio 1998. 52 Estos ejemplos están tomados del informe del Banco Inter-Armericano de Desarrollo, América Latina frente a la desigualdad. Progreso económico y social en América Latina. Informe 1998-1999, Washington, DC, BID, 1998, pp. 57, 109, 142 y s.
} 
verdadera desbandada en los grupos de mayores ingresos entre los cuales sólo el $40 \%$ e incluso, en algunos países, sólo el $25 \%$ de los niños asiste a las escuelas públicas. Existen exit forzados en algunos casos, como los provocados por las crisis financieras, los shocks macroeconómicos provocando una gran pérdida de capital humano al verse los jóvenes obligados a incorporarse en el mercado laboral antes de tiempo (exit anticipado de los estudiosos), o los trabajadores a sufrir despedidas privando las empresas de capital de conocimiento acumulado (exit del mercado laboral)..$^{53}$

Aquí, como en los ejemplos señalados anteriormente, un aspecto central consiste en priorizar los mecanismos de voice sobre los de exit. En materia de educación, por ejemplo, pueden darse mayores atribuciones a los beneficiarios proporcionándoles oportunidades para dar voz y hacer conocer su descontento; los motivos de su insatisfacción con el sistema escolar. Los consejos escolares, creados en El Salvador, Nicaragua, Brasil y Bolivia, son un ejemplo de respuesta posible, al asegurar esos consejos la participación de los padres mediante una responsabilización que va de la elección de los directores al manejo de fondos, pasando por mecanismos de evaluación de los maestros. De manera idéntica se pueden imaginar en el nivel de la educación superior sistemas participativos que involucran a los estudiantes, en particular en lo que se refiere a la evaluación de los profesores y programas propuestos, tomando una serie de precauciones para no «autosubvertir» involuntariamente el propósito de estos mecanismos evaluativos.

El análisis podría autosubvertirse señalando que en muchos casos el problema no es reducir la salida sino más bien favorecer la entrada. Por ejemplo, favorecer la incorporación de los micro-empresarios a los mercados de crédito (siendo una solución posible la de los bancos de micro-créditos) o la de las mujeres a los mercados laborales estables.

s3 Ver el estudio realizado por Gustavo Márquez, "The impact of volatily on the labor market: México, 1994-1996", Washington, DC, BID, 1998. 


\section{Conclusión}

"In dealing with the multiple and complex problems of development we have learnt that we must fashion generalizations at all kinds of ranges and be deaf, like Ulysses, to the seductive chant of the unique paradigm».

Albert Hirschman. ${ }^{54}$

Como intentamos mostrar, los trabajos de Hirschman autorizan a releer, a la luz de las experiencias prácticas, el camino recorrido y por recorrer, La distribución del ingreso y la riqueza es muy desigual en América Latina como señala el informe de la BID que invita a aprovechar la ventana de oportunidades demográficas que se presentan hoy para acelerar el desarrollo.

Sin embargo, los logros realizados en la región a nivel de su transformación estructural contrastan con el desigual avance en la resolución de estas disparidades. Las reformas estructurales llevadas a cabo en la región consiguieron poner los relojes de los países latinoamericanos a la hora del mundo mundial. Muchos de los mismos latinoamericanos siguen careciendo de los más mínimos instrumentos que les permitirían gozar de sincronización de los relojes del mundo. Carecen sencillamente de los relojes básicos, de educación, de salud, de ingresos. Los años noventa fueron años de sorprendentes logros estructurales, de reformas de gran amplitud, fueron también años de crisis brutales, de tasas de crecimiento que no se han traducido en términos idénticos para todos los sectores de las poblaciones del continente. Lo sorprendente ha sido finalmente la tolerancia de estas poblaciones cuya situación no fue mejorando.

Quizás, encontramos aquí una ilustración más del «efecto túnel» con el cual queremos acabar: mientras uno permanece en el túnel del subdesarrollo y mientras puede tener razones para esperar ver el final del túnel, porque sabe que alguno de los viajeros lo consiguió, pasó de las clases económicas a las business class, mientras existan mecanismos de ascenso social, el efecto o túnel funciona, la tolerancia hacia las desigualdades puede ser grande. Pero puede que la capacidad de aguanta se erosione, que el túnel nunca llegue al final, que se dejen de conocer traspasos de las clases económicas a las business class. «As long as the tunnel effect lasts, everybody feels better off, es-

${ }^{54}$ Albert Hirschman, A propensity to self-subersion, Cambridge, Mass., Harvard University Press, 1995, p. 116. 
cribe Hirschman, both those who have become richer and those who have not». «But this tolerance is like a credit thal falls due at a certain date. 1t is extended in the expectation that eventually the disparities will narrow again. If this does not occur, there is bound to be trouble, and, perhaps, disaster ${ }^{55}$.

Puede que el efecto túnel se esté agotando en América Latina. Puede también que esto no conduzca al desastre. Pero, para ello, una vez más habrá que inventar e imaginar, traspasos y autosubversiones, seguir indagando, con curiosidad y humildad, a imagen de Ulises, olvidando el canto majestuoso de las sirenas. Para ello no bastará ponerse cera en los oídos, sino también quizás a bias for hope en el corazón y a little more reverence for life en la mente. 